\title{
Einzelgewichte und Gewichtserträge von Schollen der Deutschen Bucht
}

\author{
Von Johannes Lundbeck \\ Bundesanstalt für Fischerei, Biologische Anstalt Helgoland, Abteilung für Fischereiforschung. \\ (Mit 6 Abbildungen im Text)
}

Den nachfolgenden Darlegungen liegt der Gedanke zugrunde, daß es bei der Scholle in ganz besonderem Maße notwendig ist, die Körpergewichte in den $\mathrm{Kreis}$ der Untersuchungen einzubeziehen. Dieser Fisch ist seit Jahrzehnten das bevorzugte Objekt der deutschen Meeresforschung, insbesondere der Biologischen Anstalt Helgoland gewesen, und es hat sich gezeigt, daß bei ihr die ökologischen Außenfaktoren aus verschiedenen Gründen besonders wechselvoll und einflußreich sind. Einmal bestehen zeitlich wie räumlich erhebliche Ungleichheiten des Wachstums, die offenbar sowohl von der Bestandsdichte wie von der Menge der verfügbaren Nahrung abhängen. Zum anderen staffeln sich die Alters- und Größengruppen nach Tiefenzonen, wobei die jahreszeitlichen Wanderungen zur Küste und wieder seewärts die Verteilung weiter komplizieren. Es besteht also hier in besonderem Maße die Möglichkeit, die Wechselbeziehungen zwischen Körperverfassung und Lebensbedingungen zu untersuchen. Allerdings bieten sich praktisch auch ungewöhnlich große Schwierigkeiten.

In einer allgemeinen Darstellung der Gewichtsverhältnisse bei unseren Nutzfischen') habe-ich die Auffassung vertreten und begründet, daß das Längen-Gewichts-Verhältnis weder unveränderlich sei noch sich nach einfachen, mathematisch faßbaren Regeln verändere, vielmehr mannigfachen, sowohl physiologisch wie ökologisch bedingten Schwankungen unterliegt. Auf die besonders starke Veränderlichkeit bei der Scholle habe ich dabei hingewiesen. Eine nähere Prüfung schien mir bei dieser erwünscht und wurde ermöglicht durch einige Reihen von Gewichtsbestimmungen bei Jungschollen aus dem Lister Wattengebiet, für deren Überlassung ich Herrn Prof. Dr. HaGmeier und Herrn Techn. Assist. A. Holtmann, ferner einige weitere von den Terminfahrten des Forschungskutters "Uthörn" in die Deutsche Bucht, für die ich den Herren Dr. Kotthaus und Dr. Ziegelmeier zu Dank verpflichtet bin. Dieses Material reicht allerdings nicht aus, die auftretenden Fragen befriedigend zu lösen, aber es kann einige wichtige Hinweise geben, die für die Biologie der Scholle von Bedeutung sind und vielleicht auch zur Weiterarbeit anregen. Außerdem wird versucht, zahlenmäßige Unterlagen für die Umrech-

1) LUNDBECK, Biologisch-statistische Untersuchungen über die deutsche Hochseefischerei III Das Körpergewicht und das Längengewidhts-Verhältnis bei den Nutzfischen. Ber., NF, 3, 1951 Dort auch Angaben über einschlägige Literatur, u. a. für die Scholle. 
nung von Stückzahlen und Länge auf Gewichte sowohl für die wissenschaftlichen Fänge wie für die Marktanlandungen zu gewinnen.

\section{Schwankungen des Verhältnisses $z$ wischen Länge und Gewicht im natürlichen Schollenbestande}

In Tabelle 1 sind zunächst die mittleren Stückgewichte je 1-cm-Längengruppe zusammengestellt. Zugrunde liegen 1531 Schollen aus dem Lister Wattenmeer 1949-1951 (gleiche Längen gemeinsam gemessen), 504 Schollen aus den Fängen der "Uthörn" zuzüglich einer Probe untermassiger Beifangschollen aus einem Kutterfang 1948-1950; dazu sind 437 Schollen aus vergleichbaren Marktfängen von der südlichen Nordsee gestellt. Um hinreichend sichere Durchschnittszahlen zu gewinnen, sind jeweils mehrere Fänge zusammengezogen; auch so noch ist die Vergleichbarkeit beschränkt, da das Material aus verschiedenen Jahren stammt.

Nur die "Uthörn"-Fänge zeigen einen ziemlich klaren jahreszeitlichen Unterschied: Im Mittel der Länge von 12 bis $23 \mathrm{~cm}$ ist das Stückgewicht der Schollen im Herbst 32 \% größer als im Frühjahr. Die Proben von List ergeben für die einzelnen Jahre unsichere (durch Interpolation ergänzte) Durchschnittsgewichte, die mir bedeutungsvoll erscheinen:

\begin{tabular}{|c|c|c|c|c|c|c|}
\hline \multirow{2}{*}{$\begin{array}{c}\text { Länge } \\
\mathrm{cm}\end{array}$} & \multicolumn{3}{|c|}{ Juni-Juli } & \multicolumn{3}{|c|}{ August-September (Oktober) } \\
\hline & 1949 & 1950 & 1951 & 1949 & 1950 & 1951 \\
\hline $\begin{array}{l}3-5 \\
6-10\end{array}$ & $\begin{array}{l}2,0 \mathrm{~g} \\
7,2 \mathrm{~g}\end{array}$ & $\begin{array}{l}0,8 \mathrm{~g} \\
4,8 \mathrm{~g}\end{array}$ & $\begin{array}{l}1,0 \mathrm{~g} \\
5,8 \mathrm{~g}\end{array}$ & $(\overline{6,3} \mathrm{~g})$ & $5, \overline{2 g}$ & $6, \overline{8}$ \\
\hline $\begin{array}{l}11-15 \\
16-20\end{array}$ & $\begin{array}{ll}28 & \mathrm{~g} \\
53 & \mathrm{~g}\end{array}$ & $\begin{array}{ll}22 & \mathrm{~g} \\
60 & \mathrm{~g}\end{array}$ & $\begin{array}{ll}26 & \mathrm{~g} \\
62 \mathrm{~g}\end{array}$ & $\left.\begin{array}{cc}28 & \mathrm{~g} \\
(58 & \mathrm{g}\end{array}\right)$ & $\begin{array}{l}22 \\
(56\end{array}$ & $\begin{array}{l}26 \\
62\end{array}$ \\
\hline
\end{tabular}

Abgesehen von einem durchschnittlich (aber nicht für jeden Fall) etwas größeren relativen ( $d, h$. auf eine bestimmte Länge bezogen) Gewicht im Herbst zeigt sich, besonders im Juni-Juli, daß 1949 die Jungschollen bedeutend schwerer waren als 1950, während das Jahr 1951 dazwischenliegende Zahlen aufweist. Da die kleinsten Längen sicher der 0-Gruppe angehören, bezieht sich die Verschiedenheit vorzugsweise auf diese, während bei den größeren Tieren geringere und andersartige Unterschiede vorhanden sind. Es steht nun fest, daß im Jahre 1950 die Menge der Schollenbrut weitaus am größten war, besonders im Vergleich zu 1949; und wir können daraus den Schluß ziehen, daß schon bei der jüngsten Brut das relative Gewicht von der Bestandsdichte abhängt. Leider liegen keine $W$ achstumsbestimmungen vor, die zum Vergleich herangezogen werden könnten und den wirklichen, d.h. Gewichtszuwachs zu berechnen gestatten.

Als ein für alle Größen vergleichbares, zugleich feineres Maß für das Verhältnis zwischen Länge und Gewicht wird weiterhin zweckmäßig der Längen-Gewichts-Koeffizient benutzt in dem Sinne, wie ihn HeINCKE eingeführt hat. Er gibt nach der Formel Gewicht $=K$. Länge ${ }^{3}$ die Abweichung des beobachteten Gewichtes in Gramm von dem Kubus der Länge (in Zentimetern) an. Zweifellos ist es nicht berechtigt, für die Scholle allgemein den Koeffizienten $1 \mathrm{zu}$ benutzen; damit erreicht man nur eine bestenfalls grobe Annäherung, die nur vereinzelt oder zufällig wirklich zutrifft. Die Schwankungen dieses Koeffizienten aber sind zweifellos geeignet, bestimmte, vor allem ökologisch begründete Ungleichheiten in der Körperverfassung der Scholle aufzudedken. 
In Abb. 1 sind zunächst die aus den Marktuntersuchungen und einigen Literaturangaben zusammengestellten Kurven der Verhältnisse zwischen der Länge und dem Längen-Gewichts-Koeffizienten der Scholle mit denjenigen aus dem neuen Material vereinigt, alles berechnet für 5-cm-Längengruppen. Bei aller Verschiedenheit im einzelnen stimmen sie sämtlich in ihrem V-oder U-förmigen Verlauf überein. Nur die Endstücke der Kurven nach rechts sind unzuverlässig, da die wenigen zugrundeliegenden Einzelgewichte der größeren Schollen sehr große Verschiedenheiten aufweisen - wie auch bei anderen Fischen, was vielleicht als eine Art Alterserscheinung aus der starken physio-

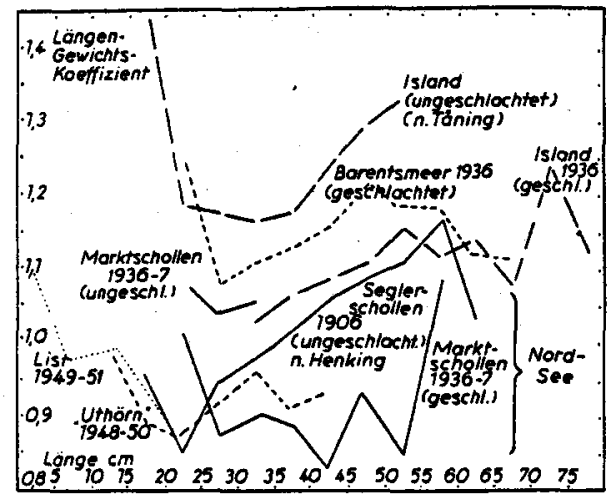

Abb. 1. Mittlere Längen-Gewichts-Koeffizienten von Schollen nach Marktuntersuchungen und Literaturangaben, für je $5 \mathrm{~cm}$ Längen berechnet

logischen Belastung durch den Aufbau der Gonaden bzw. aus dem Aufhören oder zeitweisen Aussetzen der Entwicklung zur Laichreife herrührt. Es war schon von vornherein besonders auffällig, daß der Verlauf der Kurven für die Nordsee in den Jahren 1906 und 1936 sehr verschieden war. Wie sich nun zeigt, schmiegen sich die Kurven für das Lister Wattenmeer und für die "Uthörn“-Fänge derjenigen der Seglerschollen von 1906 ziemlich eng an; es bestätigt sich damit die Vermutung, daß die damaligen Seglerfänge aus flacherem Wasser stammten als die der Dampfer und Kutter im Jahre 1936. Im letzten Falle handelt es sich auch um geschlachtete Schollen, während nur wenige ungeschlachtete "Bünn"- oder "Eisschollen" berücksichtigt werden konnten. Für einen richtigen Vergleich müßte man sich also die untere Kurve der Marktschollen von 1936 nach oben verschoben denken, so daß sie mit derjenigen der ungeschlachteten Schollen zur Dedkung kommt. Alsdann wäre das Ergebnis etwa folgendes: Der Längen-Gewichts-Koeffizient sinkt von den kleinsten Längen bis zu einem Minimum zwischen 20 und $25 \mathrm{~cm}$ Länge, um alsdann wieder zu steigen. Bei den in größeren Tiefen der Nordsee gefangenen Marktschollen liegt der Längen-Gewichts-Koeffizient bedeutend höher, erreicht sein Minimum erst bei $25-30 \mathrm{~cm}$ Länge und sinkt überhaupt nur so wenig, daß ab $25 \mathrm{~cm}$ die Kurve angenähert horizontal verläuft. Einen gleich hohen Koeffizienten erreichen die Schollen geringerer Wassertiefe nicht (s. "Uthörn"Kurve) oder erst nach der Abwanderung tiefenwärts ab etwa $40 \mathrm{~cm}$ Länge (Kurve für 1906).

Da es sich hier nun um Kombinationen je mehrerer Messungsreihen, also in sich nicht einheitliches Material handelt, ist in Abb. 2 eine möglichst weitgehende Aufteilung vorgenommen und der Kurvenverlauf des Koeffizienten 
nach 1-cm-Gruppen gezeichnet worden. Bei naturgemäß sehr viel größerer Unruhe offenbaren sich über die soeben getroffenen Feststellungen hinaus noch einige weitere Erscheinungen: Ein Gipfelwert bei etwa $5 \mathrm{~cm}$, gefolgt von einem Minimum bei $9 \mathrm{~cm}$, dann vor allem ein hoher und offenbar recht regelmäßiger Gipfel bei $14-15 \mathrm{~cm}$ mit wieder nachfolgendem Absinken. Die größeren Schollen der vergleichbaren Reihe zeigen bei ziemlicher Verschiedenheit nach dem Tiefwert unter und um $30 \mathrm{~cm}$ eine gewisse Tendenz zum Steigen. Daß es sich bei den jungen Schollen um einander abwechselnde Stadien

Tabelle 1

Mittlere Gewichte in Gramm der 1-cm-Längengruppen der Scholle

\begin{tabular}{|c|c|c|c|c|}
\hline $\begin{array}{c}\text { Länge, } \\
\text { volle } \\
\text { Zentimeter }\end{array}$ & $\begin{array}{c}\text { List } \\
\text { Sommer-Herbst } \\
1949-1951\end{array}$ & Frühl. & $\begin{array}{l}\text { Uthörn" } \\
\text { 48-1950 } \\
\text { Sommer-Herbst }\end{array}$ & $\begin{array}{l}\text { Marktfänge } \\
\text { Südl. Nordsee } 1937 \\
\text { (geschlachtet) }\end{array}$ \\
\hline 3 & (1) & - & - & - \\
\hline 4 & (1) & - & - & - \\
\hline 5 & 2 & - & - & - \\
\hline 6 & 3 & - & - & - \\
\hline 7 & 4 & - & - & - \\
\hline 8 & 5 & - & - & - \\
\hline 9 & 8 & - & - & - \\
\hline 10 & 10 & - & 15 & - \\
\hline 11 & 13 & - & 18 & - \\
\hline 12 & 19 & 18 & 20 & - \\
\hline 13 & 26 & - & 35 & - \\
\hline 14 & 31 & 30 & 40 & - \\
\hline 15 & 35 & 35 & 44 & - \\
\hline 16 & 42 & 40 & 50 & - \\
\hline 17 & 50 & 46 & 57 & - \\
\hline 18 & 55 & 54 & 64 & - \\
\hline 19 & 68 & 62 & 78 & - \\
\hline 20 & 68 & 68 & 90 & - \\
\hline 21 & 73 & 73 & 105 & 126 \\
\hline 22 & 88 & 88 & 120 & 129 \\
\hline 23 & 98 & 98 & 128 & 144 \\
\hline 24 & - & - & 148 & 160 \\
\hline 25 & - & - & 165 & 180 \\
\hline 26 & - & - & 189 & 190 \\
\hline 27 & - & - & 199 & 205 \\
\hline 28 & - & - & 228 & 214 \\
\hline 29 & - & - & 251 & 237 \\
\hline 30 & $\overline{-}$ & - & 278 & 262 \\
\hline 31 & - & - & 309 & 307 \\
\hline 32 & - & - & 370 & 320 \\
\hline 33 & - & - & 387 & 353 \\
\hline 34 & - & - & 400 & 360 \\
\hline 35 & - & - & 440 & 420 \\
\hline 36 & - & - & - & - \\
\hline 37 & - & - & - & 510 \\
\hline 38 & - & - & - & - \\
\hline 39 & - & - & - & 580 \\
\hline 40 & - & - & 620 & - \\
\hline
\end{tabular}

der Streckung und Fülle handelt, würde noch zu beweisen sein. Die Abnahme des relativen Gewichtes der heranwachsenden Schollen zwischen 15 und 25 bis $30 \mathrm{~cm}$ Länge erklärt sich vielleicht daraus, daß sie allmählich immer weniger 
zureichende Ernährungsbedingungen finden. Auch BorLEY (1912) ${ }^{2}$ ) vermutet, daß die Schollen zur Tiefenwanderung nicht nur wegen des Bevölkerungsdrudkes gezwungen werden, sondern auch wegen ihres Bedarfes an andersartiger Nahrung, d. $h$. an größeren Nahrungstieren. Auch ist anzunehmen, daß die bestgewachsenen, damit zugleich die relativ schwersten Schollen früher auf den landfernen Gründen eintreffen als die langsamwüchsigen und weniger fleischigen. $\mathrm{Ob}$ und wie der Eintritt der Geschlechts- und Laichreife das Gewicht der geschlachteten Schollen beeinflußt hat, bleibt noch zu untersuchen.

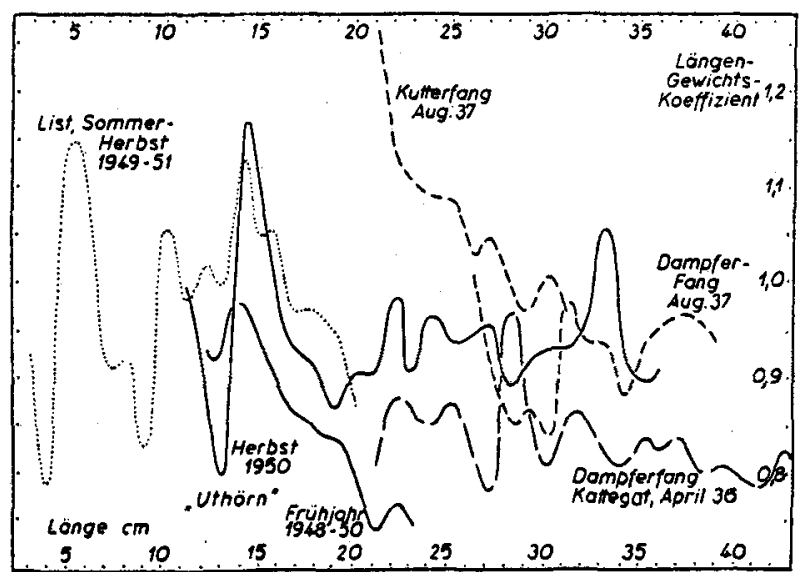

Abb. 2. Kurven der Längen-Gewichts-Koeffizienten von Schollen aus Marktanlandungen und wissenschaftlichen Fängen, berechnet für je 1-cm-Längengruppen

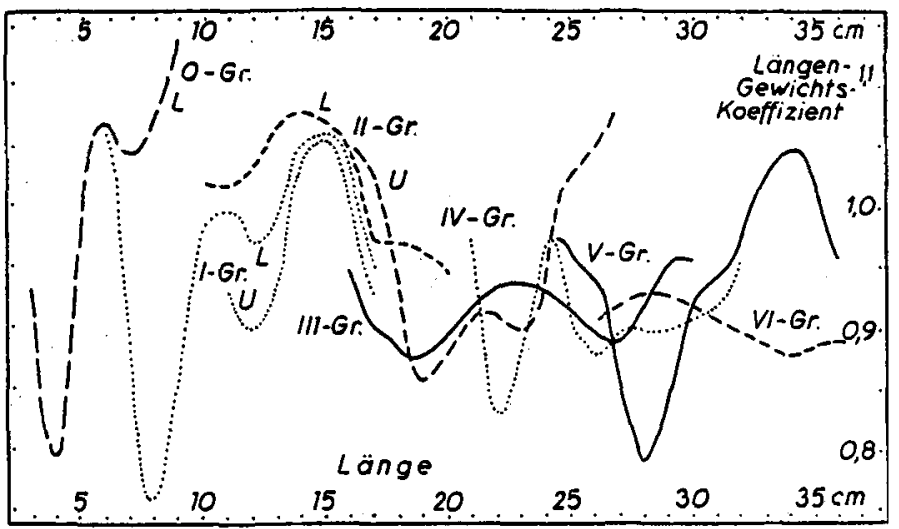

Abb. 3. Kurven der Längen-Gewichts-Koeffizienten für die einzelnen Altersgruppen aus vom Lister Wattenmeer Sommer-Herbst 1951 (" $\left.\mathrm{L}^{*}\right)$ und des Forschungskutters "Uthörn"

in der offenen Nordsee Herbst $1950\left({ }_{n} U^{\mu}\right)$, berechnet für je 1-cm-Längengruppen

Ein weiterer und sehr aufschlußreicher Einblick in die Schwankungen des Längen-Gewichts-Koeffizienten wird erreicht bei Berechnung für die einzelnen Altersgruppen, wie das in Abb. 3 für zwei Proben möglich war, diejenigen vom Sommer-Herbst 1951 aus dem Lister Wattenmeer und die von der Herbstfahrt 1950 der "Uthörn". Hier zeigt sich unverkennbar, daß auch

2) BORLEY, A Comparison of the condition of the plaice of different regions as to weight. 4. Rep. North Sea Fish. Comm. 1909 (1912). 
bei jeder Altersgruppe die Koeffizienten für die einzelnen Längen eine V- bis U-förmige Kurve ergeben. Daß mit der Wachstumsgeschwindigkeit auch das relative Gewicht allgemein steigt, habe ich schon in der genannten Arbeit nachzưweisen versucht. Daß gleichzeitig innerhalb eines bestimmten Fischbestandes bei gleicher Länge die älteren Tiere ein höheres relatives Gewicht besitzen, vermutlich wegen ungewöhnlich gedrungener Körperform, ging dort insbesondere aus einer Probe von Schollen der Deutschen Bucht hervor. Anscheinend vereinigen sich in den hier vorliegenden Kurvenbildern diese beiden Abweichungen, d. h. die besondere Fleischigkeit der längsten und die besondere Gedrungenheit der kürzeren Tiere. Die große Masse der normal gewachsenen weist also den kleinsten, gewissermaßen normalen LängenGewichts-Koeffizienten auf. Aber obwohl das Material zu geringfügig war, um beweiskräftig zu sein, ist die Kurve der Frequenzen je Längenintervall unverkennbar derjenigen des Längen-Gewichts-Koeffizienten spiegelbildlich ähnlich. Zum mindesten bei den jüngsten Altersgruppen, die in der Kurve der Längenhäufigkeit gesonderte Gipfel bilden, wäre also auch mit einem Einfluß des Nahrungswettbewerbs zu rechnen. Vielleicht kann man die starke Schwankung des Koeffizienten bei der 0- und I-Gruppe aus einer Summierung dieses Bevölkerungsdruckes mit den vorher erörterten Ungleichheiten von Körperform und -dicke erklären, während bei den älteren Klassen die Häufigkeit sich gleichmäßig über alle Längen verteilen. Uberraschend ähnlich sind übrigens die Kurven für die I- und II-Gruppe aus dem Watt und der offenen Nordsee, woraus hervorgeht, daß die Sdrwankungen im relativen Gewicht durchaus nicht zufällig sind. Daß bei diesen Kurvenpaaren die Werte für die Nordsee etwas geringer sind als für das Watt, kann man vielleicht durch die Anstrengungen der Wanderung und die Umstellung in der Lebensweise erklären.

Bei einem Vergleich der Kurven der Abb. 3 mit denen der Abb. 2 (beide auf nicht ganz gleichem Zahlenmaterial beruhend) wird deutlich, daß die $\mathrm{Ge}-$ samtkurve des Längen-Gewichts-Koeffizienten in hohem Maße von denjenigen der einzelnen Altersgruppen abhängt. Daher wird man auch mit aller Art von Veränderungen rechnen müssen, je nachdem wie stark die einzelnen Altersklassen sind und auf welche Längen sich ihre Mitglieder konzentrieren, was von der Wachstumsgeschwindigkeit, Jahreszeit usw. abhängt.

\section{Die Gewichtserträge der Schollenjahrgänge in Abhängigkeit von Wachstum und Marktauslese}

Aus dem bisher Gesagten geht hervor, daß das relative Gewicht in jedem Jahr, für jede Altersklasse und Länge, ferner auch nach Jahreszeiten und Gebieten verschieden sein kann. Aus diesem Grunde ist es so gut wie unmöglich, für die Umrechnung der Schollen bestimmter Längen allgemein gültige Stückgewichte anzugeben. Für die Kutterfänge der dreißiger Jahre sind diejenigen der damals gewogenen Marktschollen zugrunde gelegt worden. Diese sind in Tabelle 2 zusammengestellt.

Besonders wichtig ist es nun aber festzustellen, welche Fangausbeuten die einzelnen Altersklassen der praktischen Fischerei gewährt haben und wie sich diese auf die verschiedenen Altersstadien verteilen. Damit ist alsdann die Möglichkeit gegeben, Vergleiche mit der Stärke der Jahrgänge und mit ihrer Wachstumsgeschwindigkeit anzustellen. Zahlenmäßig ist ein solcher Vergleich 
möglich durch Gegenüberstellung der laufenden Bestandskontrollen durch Bückmann, Kotthaus und Schmidt mit den Analysen der Marktanlandungen und den Ertragsstatistiken der Hochseekutter von LundBEcK und SCHMIDT ${ }^{3}$ ); aber in praktischer Hinsicht ausschlaggebend sind die Gewichtsmengen.

Tabelle 2

Durchschnittliche Stüdkgewichte der Marktschollen aus der Nordsee 1936-1937, berechnet aus den geglätteten Längen-Gewichts-Koeffizienten.

\begin{tabular}{|c|c|c|c|c|c|c|c|}
\hline $\begin{array}{l}\text { Länge } \\
1 \text { cm- } \\
\text { Gruppe }\end{array}$ & $\begin{array}{c}\text { 1/100 } \\
\text { Kubus d. } \\
\text { Länge }\end{array}$ & $\begin{array}{l}\text { Längen- } \\
\text { Gewichts- } \\
\text { Koeffizient }\end{array}$ & $\begin{array}{l}\text { Stück- } \\
\text { gewicht } \\
\text { Gramm }\end{array}$ & $\begin{array}{c}\text { Länge } \\
1 \text { cm- } \\
\text { Gruppe }\end{array}$ & $\begin{array}{c}\text { 1/100 } \\
\text { Kubus d. } \\
\text { Länge }\end{array}$ & $\begin{array}{l}\text { Längen- } \\
\text { Gewichts- } \\
\text { Koeffizient }\end{array}$ & $\begin{array}{l}\text { Stück- } \\
\text { gewicht } \\
\text { Gramm }\end{array}$ \\
\hline 19 & 74 & 1,12 & 84 & 35 & 447 & 0,94 & 420 \\
\hline 20 & 86 & 1,11 & 95 & 36 & 486 & 0,94 & 457 \\
\hline 21 & 99 & 1,10 & 109 & 37 & 527 & 0.93 & 490 \\
\hline 22 & 114 & 1,08 & 123 & 38 & 571 & 0,92 & 526 \\
\hline 23 & 130 & 1,06 & 138 & 39 & 616 & 0,91 & 560 \\
\hline 24 & 147 & 1,02 & 150 & 40 & 664 & 0,89 & 592 \\
\hline 25 & 166 & 0,98 & 163 & 41 & 715 & 0,88 & 628 \\
\hline 26 & 186 & 0,91 & 170 & 42 & 768 & 0,87 & 666 \\
\hline 27 & 208 & 0,84 & 174 & 43 & 823 & 0,86 & 708 \\
\hline 28 & 231 & 0,83 & 192 & 44 & 881 & 0,87 & 765 \\
\hline 29 & 257 & 0,83 & 213 & 45 & 942 & 0,89 & 840 \\
\hline 80 & 284 & 0,85 & 241 & 46 & 1005 & 0,93 & 935 \\
\hline 31 & 313 & 0,88 & 275 & 47 & 1072 & 0,96 & 1030 \\
\hline 32 & 343 & 0,91 & 312 & 48 & 1141 & 0,98 & 1120 \\
\hline 33 & 376 & 0,93 & 349 & 49 & 1213 & 0,98 & 1190 \\
\hline 34 & 411 & 0,94 & 386 & 50 & 1288 & 0,97 & 1250 \\
\hline
\end{tabular}

Eine Umrechnung wird am genauesten, wenn man die Längenzusammensetzung der einzelnen Altersklassen zugrunde legt. Ein einfacheres Verfahren geht von der mittleren Länge der Altersklassen aus, indem man das diesen entsprechende durchschnittliche Stückgewicht ermittelt und einfach mit der Stückzahl multipliziert. Dabei begeht man aber einen Fehler: $\mathrm{Da}$ das Gewicht als dreidimensionale Größe schneller wächst als die Länge, werden die hohen Gewichte der längsten Tiere nicht genügend einbezogen, das Gewichtsmittel fällt also zu niedrig aus. In Tabelle 3 sind für zwei Jahre die Ergebnisse beider Berechnungsweisen untereinandergestellt; es zeigt sich, daß bei dem zweiten Verfahren bis $8 \%$ zu niedrige Gewichte in Anrechnung kommen. Bei den jüngsten Jahrgängen tritt dieser Fehler nicht auf oder verkehrt sich sogar in das Gegenteil; das beruht neben einer geringen Größenvariation vermutlich darauf, daß diese Altersgruppen nur mit einer kleinen Zahl der Bestgewachsenen in den Marktfängen vertreten sind, überdies im Bereich der betreffenden Längen der Längen-Gewichts-Koeffizient abnimmt. Im allgemeinen wird man also damit zu rechnen haben, daß die älteren Jahrgänge im Vergleich zu den jüngsten etwas zu niedrig angesetzt werden.

Die mittleren Längen der Altersgruppen in den Anlandungen und damit auch die Durchschnittsgewichte werden nun nicht allein oder in erster Linie durch das Wachstum selbst, d. h. durch die wirkliche Größe der Jahrgänge in den Beständen bestimmt, sondern vielmehr durch die Netz- und Marktauslese.

3) Siehe Ber. Deutsch. Wiss. Komm. f. Meeresf., N. F. 6 u. 7 (1932-34) und „Fischmarkt ${ }^{*}$ 1934-38. 
Angaben über die erste machte BÜCKMANN ${ }^{4}$ ); aber seine Feststellungen können nicht sicher und uneingeschränkt als für die gewerbliche Fischerei gültig betrachtet werden. Ubrigens spielt die Netzauslese für die Größenzusammensetzung der Anlandungen und die der Altersgruppen in ihnen eine geringere Rolle als die Marktauslese. Eine Analyse der Netzfänge könnte nur auf weniger geeigneter Grundlage die mit Hilfe wissenschaftlicher Fänge durchgeführte Bestandesüberwachung wiederholen; ihr hauptsächlicher und in dieser Hinsicht allerdings recht großer Wert besteht vielmehr in der zahlenmäßigen Feststellung der Marktauslese. Durch diese werden die kleinsten Schollen der Netzfänge bis zu einer bestimmten Grenze von der Anlandung ausgeschlossen. Nach meinen früheren Angaben wurde 1930-1931 von den $20 \mathrm{~cm}$ langen Schollen $0,1 \%$ verwertet und dann mit steigendem Anteil bis zu den restlos angelandeten $29 \mathrm{~cm}$ langen; das bedeutet für die Altersgruppe II 0,5\% Marktfähige und weiter zunehmend bis zur vollständigen angelandeten VII-Gruppe. Von jeder Altersgruppe kommen also nur diejenigen Schollen an den Markt, die bereits marktfähige Größe erreicht haben. Ihre Zahl ist um so kleiner, je weniger sie in ihrer Größenzusammensetzung die Grenze der Marktfähigkeit

Tabelle 3

Mittlere Längen und Gewichte der Altersklassen in den wissenschaftlichen Fängen und in den Anlandungen der Scholle

\begin{tabular}{|c|c|c|c|c|c|c|c|}
\hline Altersgruppe & II & III & IV & $\mathrm{V}$ & VI & VII & VIII \\
\hline \multicolumn{8}{|c|}{ Fangjahr $1930:$} \\
\hline \multicolumn{8}{|l|}{ Mittlere Längen in Zentimetern: } \\
\hline Wissenschaftliche Fänge & 13,1 & 19,3 & 23,8 & 26,4 & 28,9 & 34,2 & - \\
\hline Marktanlandungen & 23,8 & 25,0 & 26,1 & 27,5 & 29,3 & 31,2 & 31,8 \\
\hline \multicolumn{8}{|l|}{$\begin{array}{l}\text { Mittlere Gewichte in den } \\
\text { Anlandungen in Gramm: }\end{array}$} \\
\hline A. d. Summe d. Einzelgew. & 142 & 154 & 169 & 187 & 227 & 280 & 301 \\
\hline A. d. Längenmittel ber. & 143 & 154 & 163 & 174 & 207 & 267 & 282 \\
\hline \multirow[t]{2}{*}{$\%$-Abweichung } & +1 & \pm 0 & -4 & -7 & -8 & -5 & -6 \\
\hline & \multicolumn{7}{|c|}{ Fangjahr 1931: } \\
\hline \multicolumn{8}{|l|}{ Mittlere. Längen in Zentimetern: } \\
\hline Wissenschaftliche Fänge & 13,1 & 16,6 & 22,5 & 26,2 & 29,2 & 34,2 & - \\
\hline Marktanlandungen & 25,4 & 24,8 & 26,2 & 27,5 & 28,5 & 30,0 & 29,7 \\
\hline $\begin{array}{l}\text { Mittlere Gewichte in den } \\
\text { Anlandungen in Gramm: }\end{array}$ & & & & & & & \\
\hline A. d. Summe d. Einzelgew. & 158 & 152 & 163 & 188 & 204 & 239 & 229 \\
\hline A. d. Längenmittel ber. & 161 & 156 & 164 & 174 & 192 & 233 & 217 \\
\hline$\%$-Abweichung & +2 & +3 & +1 & -7 & -6 & -3 & -5 \\
\hline
\end{tabular}

überschreiten. Die mittlere Größe in den Anlandungen ist also höher als in den unausgelesenen Netzfängen und insbesondere in den u. a. infolge Verwendung von „Dedksteerten“ schärfer die kleinen Tiere erfassenden der Bestandsuntersuchungen. Der Unterschied ist um so stärker, je geringer die mittlere Größe ist. Auch darüber gibt Tabelle 3 Auskunft.

Nun ist die Marktauslese nicht unveränderlich. Wenn man den Scheidewert als Vergleichsmaß setzt, d. h. die Länge, bei der gerade die Hälfte der

4) Bückmann: Die Frage nach der Zweckmäßigkeit des Schutzes untermassiger Fische... Rapp. Pr. Verb. 80, 1932. 
gefangenen Schollen als marktfähig ausgelesen wird, so veränderte sich dieser im Laufe der Jahre in folgender Weise:

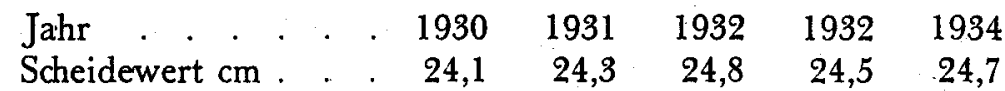

Für die weiteren Jahre liegen entsprechende Angaben leider nicht mehr vor. 1930-1934 wurde nach diesen Zahlen also die Marktauslese verschärft, d. h. es wurde ein abnehmender Teil der kleinsten mitgefangenen Schollen verwertet. Die Ursache dafür ist in den Absatzbedingungen zu suchen, die im Jahre 1932 als Folge der allgemeinen Wirtschaftskrise besonders schwierig waren. Daß schon die Heraufsetzung des Scheidewertes um wenige Millimeter erhebliche praktische Bedeutung besitzt, habe ich damals nachzuweisen versucht: Bei der Abnahme des Schollenfanges unserer Hochseekutter 1930-1932, die sich auf $30 \%$ der Stückzahl und $20 \%$ des Gewichtes belief, fiel der kleinere Teil, nach meinen Berechnungen rund 1 Million Schollen mit 1000 Zentnern Gewicht, infolge schlechten Wachstums, also verzögerter Erreichung marktfähiger Größe, aus, dagegen rund doppelt so viel durch die verschärfte Marktauslese.

Die tatsächliche (durch die wissenschaftlichen Fänge festgestellte) mittlere Größe der Altersklassen ist für diejenige in den Marktanlandungen nur von untergeordneter Bedeutung. Das sollen die Kurven der Abb. 4 darstellen: Von 1930 auf 1931 verschlechterte sich das Wachstum der Scholle, aber trotzdem waren die jüngeren Altersklassen in den Kutterfängen durchschnittlich größer, weil sie schärfer ausgelesen waren. Mit weiter verschärfter Marktauslese hätte für 1932 eine weitere Steigerung der mittleren Größe erwartet werden sollen. Wenn das nicht der Fall war, so beruhte das auf einer weiteren Herabminderung des Wachstums, besonders bei dem reichen, damals vierjährigen Jahrgang 1928; es waren eben einfach keine oder nur sehr wenige Tiere vorhanden, welche die ihrem Alter sonst entsprechende maximale Größe erreicht hatten. Insofern ist also auch die Wachstumsgeschwindigkeit neben der Auslese von Einfluß. In erster Linie aber wirkt sie auf den Anteil der marktfähigen Tiere, der für die Dreijährigen 1932 nur mehr $0,8 \%$ betrug gegen $8,5 \%$ ein und $12,9 \%$ zwei Jahre vorher.

Im allgemeinen wiesen damals auch die jüngsten, in den Anlandungen vertretenen Altersgruppen kein geringeres Längenmittel als etwa $23 \mathrm{~cm}$ und kein niedrigeres für das Stückgewicht als $150 \mathrm{Gramm}$ auf. Eine mittlere Länge von 11-12 cm wird also durch die Marktauslese auf das Doppelte heraufgesetzt, eine größere entsprechend weniger, bis bei rund $30 \mathrm{~cm}$ keine Veränderung mehr eintritt. Für die Gewichtsmittel gilt genau das gleiche, aber die Erhöhung ist hier sehr viel stärker und geht bei den geringsten in Frage kommenden Gewichten bis über $500 \%$. Das Ausmaß dieser Heraufsetzung der mittleren Länge und des mittleren Gewichtes ist in Abb. 5 graphisch dargestellt; jedoch gelten die Kurven genau genommen nur für eine bestimmte Marktauslese; die eingezeichneten Zahlenwerte für 1931 liegen auch sämtlich über denen von 1930, und beide Reihen würden je eine besondere Kurve zu ziehen gestatten, während das Jahr 1932 wegen der erwähnten widerstreitenden Veränderungen weniger klare Verhältnisse zeigt.

Bei der Berechnung des Gewichtsanteiles derAltersgruppen an den Anlandungen sind erhebliche Fehler trotz des mangelhaften Unterlagenmaterials nicht zu erwarten, sofern die Längen-Gewichts-Koeffizienten der verschiedenen 
Zentimeterbereiche in richtigem Verhältnis zueinander stehen; das Gesamtgewicht steht statistisch fest, und die Verteilung nach Stückzahlen auf Alter und Längen ist durch Marktproben hinreichend sicher ermittelt. Die Summe der aus Stückzahl mal Stüdkgewicht berechneten Gesamtgewichte übersteigt diejenigen der Fischereistatistik um durchschnittlich $12 \%$, bleibt nur in einem

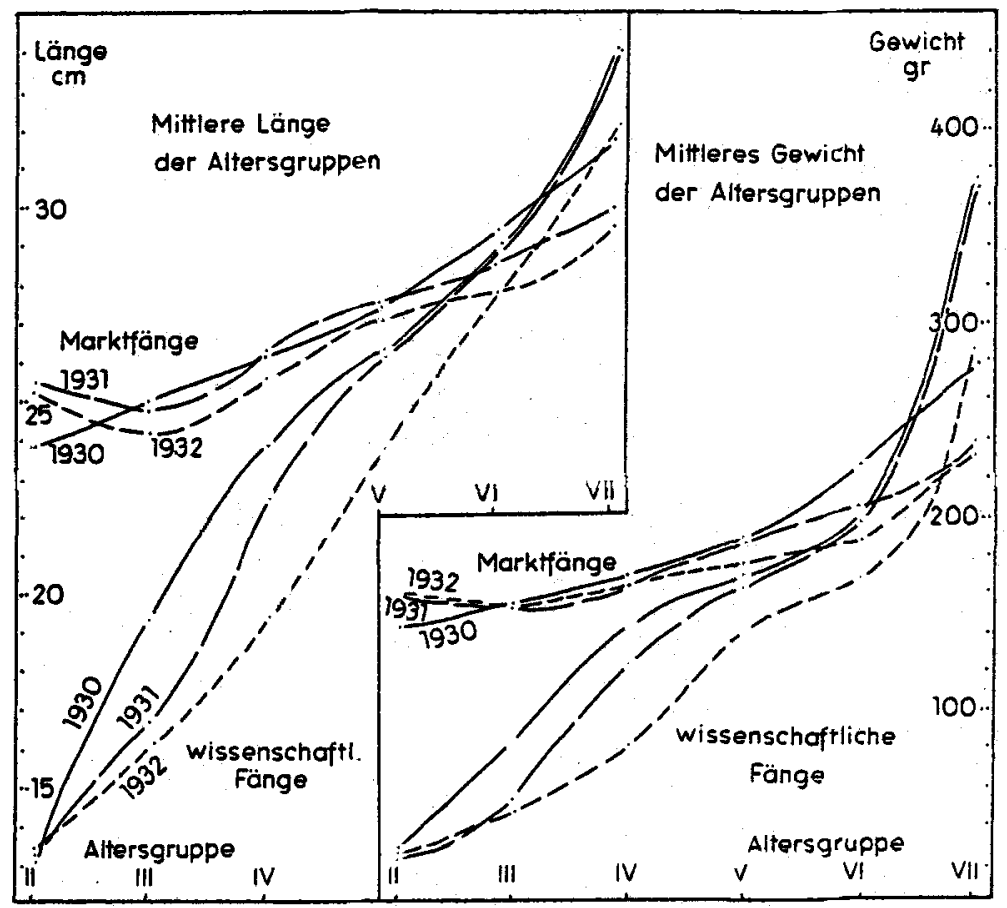

Abb. 4. Mittlere Längen (links) und Gewichte (rechts) der Schollen in den wissenschaftlichen Fängen und in den Marktanlandungen 1930-1932

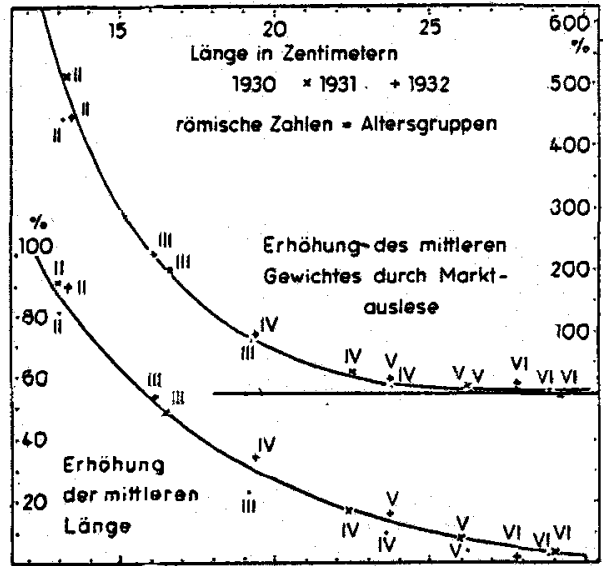

Abb. 5. Kurven für die Heraufsetzung der mittleren Länge (unten) und des mittleren Gewichtes der einzelnen Schollenjahrgänge durch die Marktauslese 1930-1932

Jahre (1932) darunter und erreicht höchstens reichlich 20\%. Diese Ubereinstimmung kann als befriedigend betrachtet werden; die etwas $2 u$ hoch berech- 
neten Stüdkgewichte erklären sich sehr wahrscheinlich daraus, daß Schollen aus den Frühjahrsmonaten nicht genügend stark beteiligt sind. Daß nicht in jedem Jahre, Gebiet und Monat besondere Gewichtsbestimmungen vorgenommen und verwendet wurden, sondern lediglich berechnete Durchschnitte, verbietet allerdings alle Erörterungen bezüglich etwaiger Schwankungen des relativen Gewichtes in Abhängigkeit von Bestandesdichte und Wachstum ebenso wie bezüglich möglicher Unterschiede zwischen Markt- und untermassigen Schollen usw.

Der gewichtsmäßige Altersaufbau der Schollenfänge unserer Hochseekutter in den 8 Untersuchungsjahren ist in Abb. 6 dargestellt. Verglichen mit

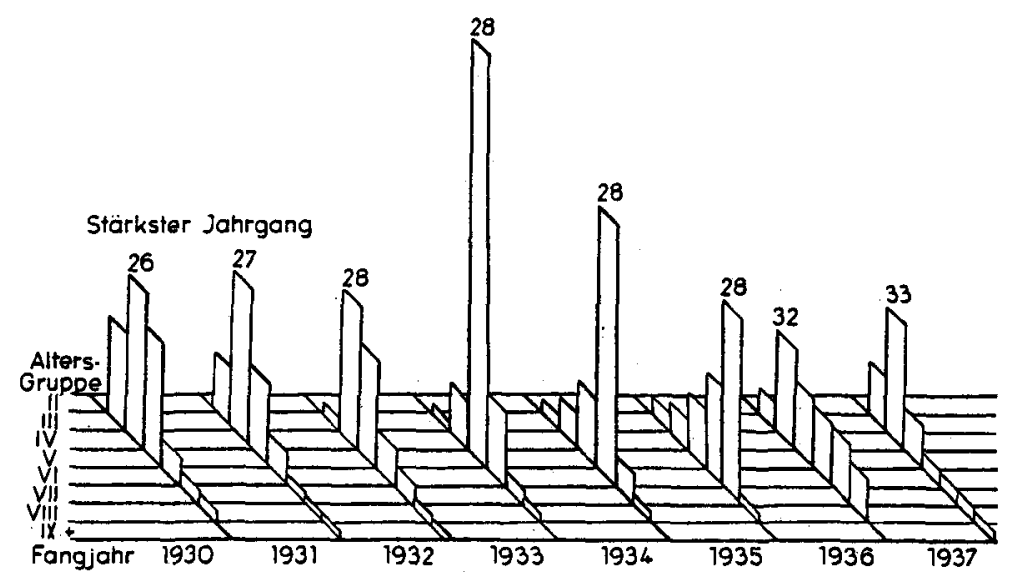

Abb. 6. Verteilung der Schollenanlandungen der deutschen Hochseekutter in den Jahren 1930-1937 auf die einzelnen Altersklassen und Jahrgänge nach Gewicht

den entsprechenden Stückzahlen ergeben sich keine grundsätzlichen Abweichungen, nur treten die älteren, größeren und daher schwereren Tiere mit dem Gewicht etwas mehr hervor. Für den ganzen Zeitraum ergibt sich ein durchschnittlicher Altersaufbau in \% wie folgt:

\begin{tabular}{lcccccccc} 
Altersgruppe & II & III & IV & V & VI & VII & VII & IX + \\
\hline nach der Anzahl & 0,3 & 9,8 & 28,2 & 33,7 & 17,9 & 8,1 & 1,6 & 0,4 \\
nach dem Gewicht & 0,2 & 8,5 & 26,1 & 31,7 & 19,9 & 10,9 & 2,2 & 0,5
\end{tabular}

Die IV- und V-Gruppe stellen also die größte Menge sowohl nach Zahl wie nach Gewicht, aber die Gewichtsanteile verschieben sich etwas auf die älteren Gruppen. Wie groß die Mengen sind, welche von den einzelnen Jahrgängen im Laufe ihres Lebens insgesamt der Fischerei geliefert worden sind, läßt sich nur für wenige Geburtsjahre ungefähr ermitteln, wie das in Tabelle 4 geschehen ist. Der Vergleich zwischen zahlenmäßiger Stärke und Nutzertrag ist entsprechend beschränkt. Man kann nur etwa folgende Verhältniszahlen einander gegenüberstellen, wobei der schwächste Tahrgang 1926 mit 1 als Grundlage gewählt ist:

\begin{tabular}{|c|c|c|c|c|c|c|}
\hline Jahrgang & & 1925 & 1926 & 1927 & 1928 & 1929 \\
\hline Stärkeverhältnis im Bestande & & 2,0 & 1,0 & 1,4 & 4,0 & 1,3 \\
\hline Verhältnis der Nutzerträge & $\left\{\begin{array}{l}\text { nach Anzahl } \\
\text { nach Gewicht }\end{array}\right.$ & $\begin{array}{l}1,6 \\
1,5\end{array}$ & $\begin{array}{l}1,0 \\
1,0\end{array}$ & $\begin{array}{l}1,2 \\
1,2\end{array}$ & $\begin{array}{l}2,6 \\
2,7\end{array}$ & $\begin{array}{l}0,7 \\
0,7\end{array}$ \\
\hline
\end{tabular}


Tabelle 4

Nutzerträge einiger Schollenjahrgänge in der Deutschen Bucht für die deutsche Kutterfischerei, in 1000 Kilogramm (in Klammern: \%)

\begin{tabular}{|c|c|c|c|c|c|c|}
\hline Geburtsjahr & 1925 & 1926 & 1927 & 1928 & 1929 & 1930 \\
\hline $\begin{array}{c}\text { Altersgruppe } \\
\text { II } \\
\text { III } \\
\text { IV } \\
\text { V } \\
\text { VI } \\
\text { VII } \\
\text { VIII } \\
\text { IX + }\end{array}$ & $\begin{array}{c}? \\
? \\
? \\
688(20) \\
198(6) \\
95(3) \\
26(1) \\
8\end{array}$ & $\begin{array}{c}? \\
? \\
871(40) \\
481(22) \\
177(8) \\
83(4) \\
23(1) \\
3\end{array}$ & $\begin{array}{c}? \\
521(20) \\
899(34) \\
603(23) \\
419(16) \\
158(6) \\
17(1) \\
0\end{array}$ & $\begin{array}{r}32 \\
310(15) \\
775(13) \\
2242(37) \\
1419(23) \\
1020(17) \\
219(4) \\
45(1)\end{array}$ & $\begin{array}{c}2 \\
22(1) \\
254(17) \\
362(24) \\
528(34) \\
309(20) \\
56(4) \\
?\end{array}$ & $\begin{array}{c}0 \\
40(4) \\
160(18) \\
308(34) \\
272(30) \\
76(8) \\
? \\
?\end{array}$ \\
\hline $\begin{array}{l}\text { ausammen } \\
\text { etwa } \\
=1000 \text { Stüdk } \\
\text { mittl. Stück- } \\
\text { gewicht in g }\end{array}$ & $\begin{array}{l}3400(100) \\
21000\end{array}$ & $\begin{array}{l}2200(100) \\
13817\end{array}$ & $\begin{array}{l}2647(100) \\
16380\end{array}$ & $\begin{array}{l}6062(100) \\
36039\end{array}$ & $\begin{array}{l}1533(100) \\
9032\end{array}$ & $\begin{array}{l}900(100) \\
5400\end{array}$ \\
\hline
\end{tabular}

Damit wird bestätigt, daß starke Jahrgänge nicht einen ihrer zahlenmäßigen Stärke voll entsprechenden höheren Nutzwert erreichen. Ihr $\mathrm{Ge}$ wichtsertrag ist demjenigen nach der Anzahl sehr ähnlich, denn bei langsamerem Wachstum werden die Schollen eben in entsprechend höherem Alter gefangen, in dem sie das marktwürdige Gewicht erreicht haben; das gilt ebenso wie für die volksstarken Jahrgänge selbst wie für diejenigen wenig jüngeren (und auch älteren), die zwar selbst schwach sind, aber unter der Wirkung der reichen Jahrgänge ebenfalls langsam wachsen. In solchen Fällen stehen sie statt mit 4 Jahren erst ein oder zwei Jahre später in wirtschaftlicher Bedeutung auf ihrem Höhepunkt. Vermutlich erklärt dies auch, weshalb sie keine so großen Ausbeuten ergeben, wie es ihrer Stärke entsprechen würde: Während der zur Erreichung martkfähiger Größe erforderlichen längeren Zeit sinkt ihre Zahl durch natürliche Sterblichkeit mehr als das bei schnellwüchsigen Jahrgängen der Fall ist. Würde große Volksstärke nicht mit vermindertem Wachstum verbunden sein, darf also wohl ein jener voll entsprechender Nutzwert vermutet werden; doch fehlen darüber noch Erfahrungen bei anderen Fischarten. Wenn sich aus Tabelle 4 ergibt, daß die langsamwüchsigen Jahrgänge Marktschollen von durchschnittlich größerem Stückgewicht erbracht haben (das Mittel aller Jahre beläuft sich auf 166 Gramm), so kann das auch allein durch die damals verschärfte Marktauslese erklärt werden. Die gleichzeitig eingetretene Intensivierung der gesamten Schollenfischerei dagegen müßte das mittlere Alter und Gewicht der Marktschollen herabgesetzt haben. Der vorliegende Fall zeigt also das Zusammenwirken verschiedener biologischer Faktoren Bestandesdichte, Wachstumsgeschwindigkeit, natürlicher und fischereilicher Abgang - mit solchen wirtschaftlicher Art - Fangintensität, Marktauslese -, wobei der Einfluß jedes einzelnen von ihnen genauer nur durch sehr eingehende Vergleichsuntersuchungen ermittelt werden könnte. 
Zusammenfassung. Der Längen-Gewichts-Koeffizient der. Schollen ist bei den kleinsten Tieren zunächst hoch, nimmt dann stark ab, um im späteren Leben erneut zu steigen. Bei gleichaltrigen Schollen sind die best- und schlechtestgewachsenen Tiere relativ schwerer als die große Masse. Langsames Wachstum und große Bestandesdichte setzen das auf eine bestimmte Länge bezogene Gewicht herab; mit der Entfernung vom Land steigt es. Die Größe der Schollen in den Marktfängen hängt außer von Alter und Wachstum entscheidend auch von der Marktauslese ab: Ist diese scharf, so ergeben sich selbst für langsam gewachsene Jahrgänge erhöhte Mittelwerte von Gewicht und Länge, die bis zum Fünffachen des Gewichts und dem $Z$ weifachen der Länge der betreffenden Altersgruppen im natürlichen Bestande erreichen. Langsames Wachstum wirkt sich in der Fisctierei mehr durch verspätet eintretende Marktgröße aus. Infolge des somit länger anhaitenden natürlichen Abganges erleidet die wirtschaftliche Ertragfähigkeit langsamwüchsiger Altersgruppen Einbußen, die trotz erhöhten Alters nicht durch größere Stückgewichte ausgeglichen werden. 